\title{
Cerebral Palsy with Nephrotic Syndrome : A Rare Association- First Case Report in Bangladesh
}

\author{
M K Alam ', L Yeasmin ${ }^{2}$, S A Begum ${ }^{3}$, A H Khan ${ }^{4}$, M U Alam ${ }^{5}$, D Hossain 6
}

\section{Introduction}

Cerebral palsy is a blanket term commonly referred to as "CP" and described by loss or impairment of motor function, cerebral palsy is actually caused by brain damage ${ }^{1}$. The brain damage is caused by hypoxic brain injury or abnormal development of the brain that occurs while a child's brain is still developing before birth, during birth, or immediately after birth². In most cases, the cause of cerebral palsy is unknown. Some possible causes are: infections during pregnancy that may damage a fetus' developing nervous system includes rubella, cytomegalovirus, and toxoplasmosis. Rh incompatibility between mother and infant leads to a form of jaundice severe enough to cause brain damage ${ }^{3}$. The most common pathological changes are periventricular leukomalacia and periventricular hemorrhagic infarction ${ }^{4}$. Symptoms of $\mathrm{CP}$ patients in infancy commonly shows abnormal posture and movement retardation and low muscle tone, then it develops into hypermyotonia. Secondary changes, such as joint contracture and deformity of the spine, and progressive developments; epilepsy, mental retardation, behavioral disorders and sensory disturbances can be found, as well. Drug therapy includes neurotonic medicine, muscle relaxant, etc. Recently neural stem cell implantation treatment effectively increase the number of brain nerve cells and also is able to start the re-development of the neurological process ${ }^{4-7}$. Nephrotic syndrome is a common childhood kidney disease characterized by protein leakage from blood to the urine through the glomeruli, resulting in massive proteinuria ( $>40 \mathrm{mg} / \mathrm{m}^{2} /$ hour), hypoalbuminemia $(<2.5 \mathrm{~g} / \mathrm{dl})$, hypercholesterolemia $(>200 \mathrm{mg} / \mathrm{dl})$ and generalized edema ${ }^{8}$. The peak incidence of nephrotic syndrome in pre-school age children, $80 \%$ of nephrotic children are less than 6 years old at presentation. Management of nephrotic syndrome is symptomatic and supportive but steroid is the mainstay of treatment ${ }^{9}$.

\section{Case Report}

\section{$2,3,4,5,6$}

Dept. of Paediatric Nephrology National Institute of Kidney Diseases \& Urology (NIKDU) Sher-e-Bangla Nagar, Dhaka

\section{Correspondence}

Dr. Md. Kabir Alam

Assistant Professor

Dept. of Paediatric Nephrology

National Institute of Kidney

Diseases \& Urology (NIKDU)

Sher-e-Bangla Nagar, Dhaka

e-mail: dr.kabiralom@gmail.com
Weight $10 \mathrm{~kg}$ \& height $73 \mathrm{~cm}$, weight for age Z score 2 \& height for age $Z$ score -4 . He had scissoring gait (Fig-1) and toe standing. He had intermittent neck control (Fig-2), cannot sit without support and his fine motor also impaired. His distraction test was normal, vision impaired and speech was delayed. His milestones of development was globally delayed. His abdominal examination showed ascities, umbilicus was everted, no organomegally, both kidneys were not ballotable and external genitalia were normal. On skin survey BCG mark present, other systems examination revealed no abnormalities.

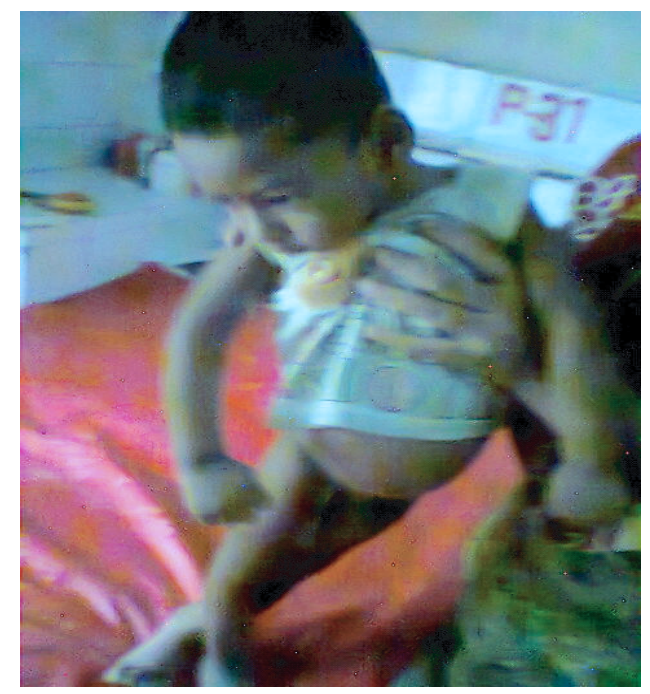

Figure-1: Scissoring posture

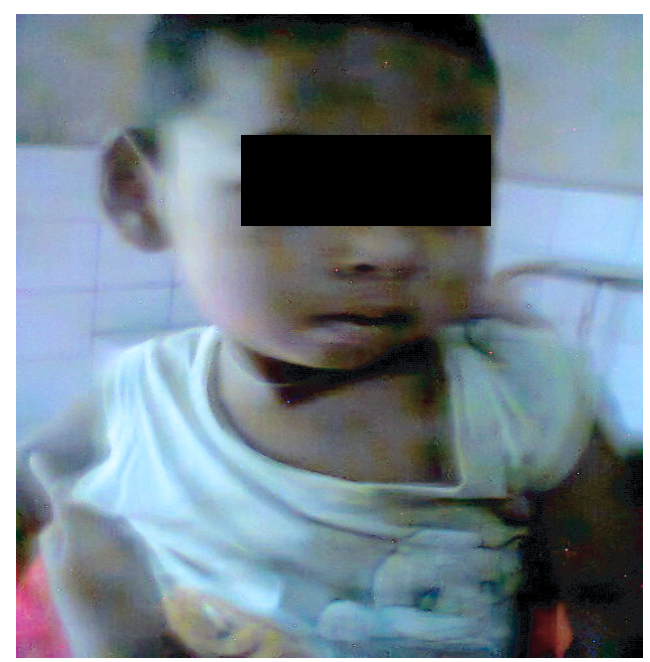

Figure-2: Intermittent neck control 


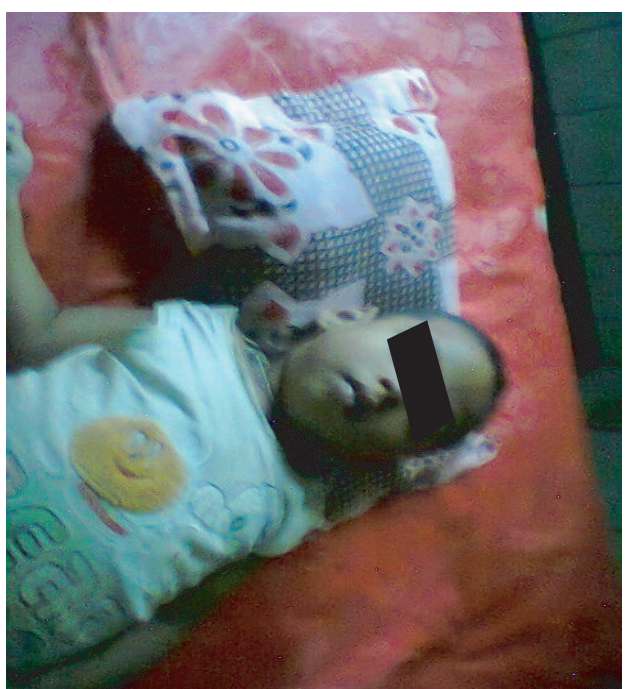

Figure-3: Microcephaly

Mother was 23 years, primi had regular antenatal check up and was normotensive \& non diabetic. The child was delivered at term in a private hospital by LUCS. As far as she remember her child had no history of delayed crying, but a generalized convulsion occurred after 3 to 4 hours postnataly. The child then managed by intravenous infusion, antibiotics, anticonvulsant and nothing per oral for ten days. She could not memorize about neonatal jaundice, condition of umbilical stump or any other event. The child was exclusively breast fed up to four months of his age and now on family diet.

Investigations showed urine routine microscopic examination albumin+++, pus cell 8-10/hpf, RBC 15-20/hpf, culture showed growth of E.Coli $\left(>10^{5} / \mathrm{ml}\right)$. Blood pictures showed hemoglobin $10.6 \mathrm{gm} / \mathrm{dl}$, ESR-35 mm in $1^{\text {st }}$ hour, WBC- $8000 / \mathrm{mm}^{3}$, neutrophil-50\%, lymphocyte$43 \%$, monocyte-03\%, eisonophil-04\%, platelet count-4,30,000/mm $\mathrm{mm}^{3}$, serum albumin $2.2 \mathrm{gm} / \mathrm{dl}$, serum cholesterol $520 \mathrm{mg} / \mathrm{dl}$, serum creatinine $0.5 \mathrm{mg} / \mathrm{dl}$, serum calcium $8.6 \mathrm{mg} / \mathrm{dl}, \mathrm{HBsAg}$ and Anti-HCV were nonreactive. Ultrasonography of Kidney-Ureter-Bladder(KUB) region showed right kidney bipolar diameter $68 \mathrm{~mm}$ and left one $70 \mathrm{~mm}$, cortical echogenicity of both kidneys were hypoechoic and corticomedullary differentiation was maintained, pelvicaliceal systems are not dilated, bladder wall was irregular. Chest X-ray showed normal findings. CT Scan of brain revealed bilateral cortical atrophy. With all his complaints, physical findings and investigations the patient was diagnosed as Cerebral Palsy with Nephritic Syndrome(1 $1^{\text {st }}$ attack).

With the introduction of appropriate antibiotic according to culture \& sensitivity of urine his urinary symptoms gradually improved and fever subsided. After that we started oral steroid (prednisolone) and continued it as the treatment protocol of first attack nephrotic syndrome. We discharged the child and counselled the guardians properly and advised to consult immediately with an paediatric neurologist or attain any 'Shishu Bikash Kendro' of Medical Colleges.

\section{Discussion}

Cerebral palsy accounts the major disability of children worldwide. On literature review, the reasons are multi originated but still perinatal asphyxia suspected the prime contributor ${ }^{2}$. Other minor offenders are intrauterine infections, congenital brain anomaly and neonatal hyperbilirubinaemia ${ }^{3}$. Cerebral palsy with nephrotic syndrome, probably this is the first case report in Bangladesh. Nephrotic syndrome is a common clinical condition in Asian children. The prevalence of minimal change nephrotic syndrome is also higher in Indian subcontinent ${ }^{10}$. The pathogenesis of nephrotic syndrome still not identified. There is a strong evidence of immune dys-regulation, mainly involving cellmediated immunity ${ }^{11}$. Joh $\mathrm{K}$ et al. reported five cases of nephrotic syndrome due to focal segmental glomerulosclerosis (FSGS) in mentally retarded children with severe infantile spasms. Four of the five children diagnosed as West syndrome, Lennox syndrome, or petit mal epilepsy also had cerebral palsy and microcephaly ${ }^{12}$. H Sano et al. described a case of microcephaly with early onset of nephrotic syndrome ${ }^{13}$. Our case the boy also has microcephaly with nephrotic syndrome starting at the early third year of life and there is no history of seizure disorder. On hospital stay, he was treated with prednisolone orally and responds well (urinary protein free for consecutive 3 days), oedema subsided and after observing diuretic phase we discharged him with proper counseling. As the child had the first attack nephrotic syndrome and steroid responder, we did not perform renal biopsy. Though, Joh $\mathrm{K}$ et al. had done renal biopsy of their cases because of the children had nephritic syndrome presented so earlier with convulsions ${ }^{12}$.

\section{References}

1. National Institute of Neurological Disorders \& Stroke(NINDS)-National Institute of Health, Disorders A-Z, Cerebral Palsy.

2. Swaiman KF. Paediatric Neurology: Principles \& Practice 4th ed. Mosby Elsevier, 2006:1098-1105

3. The ULTIMATE Resource for EVERYTHING Cerebral Palsy ${ }^{\text {TM }}$ MyChild $^{\text {TM }}-(800) 692-4453$.

4. Rosenbloom L. Diagnosis and management of cerebral palsy. Arch Dis Child 1995; 72: 350-54.

5. Bo $W$ et al. The demand for rehabilitation therapist in Beijing health organizations over the next five years. Disabilities and Rehabilitation(WHO). 2008;30:375-80.

6. Luda Stem Cells to Treat Cerebral Palsy----New hope for children with cerebral palsy-Email:luda@zhluda.net

7. Thomas DB, Franceschini N, Hogan SL, et al. Clinical and pathological characteristics of focal segmental glomerulosclerosis pathologic variants. Kidney Int. 2006;69:920-26.

8. Gordillo R, Spitzer A. The Nephrotic Syndrome. Pediatrics in Review 2009;30:94.

9. Haycock GB. Steroid responsive nephrotic syndrome. In: RJ Postlethwaite editor. Clinical Pediatric Nephrology. $3^{\text {rd }}$ ed. Oxford: Butterworth-Heinemann Ltd, 1994;210-225.

10. Sharples PM, Poulton J, White RHR. Steroid responsive nephrotic syndrome is more common in Asian. Arch DIs Child 1985;60:1014-17.

11. Mathieson PW. Immune dys-regulation in minimal change nephropathy. Nephrol Dial Transplant 2003;18 (Suppl 6):26-29.

12. Joh K, Usui N, Aizawa S, et al. Focal segmental glomerulosclerosis associated with infantile spasms in five mentally retarded children: a morphological analysis on mesangiolysis. Am J Kidney Dis. 1991;17(5):569-77.

13. H Sano, A Miyanoshita, N Watanabe, et. al. Microcephaly and early-onset nephrotic syndrome--confusion in Galloway-Mowat syndrome. Pediatric Nephrology, 1996; $9(6): 711-14$ 